\section{Case Reports in Ophthalmology}

\title{
ExPRESS Mini-Shunt as a Treatment Alternative for Medically Uncontrolled Steroid-Induced Glaucoma in a Pediatric Patient
}

\author{
Bonnie Nga Kwan Choy ${ }^{a}$ Mandy Oi Man Wong ${ }^{b}$ \\ Jonathan Cheuk Hung Chan ${ }^{a}$ Connie Hong Yee Lai ${ }^{c}$ \\ Jimmy Shiu Ming Lai \\ ${ }^{a}$ Department of Ophthalmology, The University of Hong Kong, Hong Kong, PR China; \\ ${ }^{b}$ Department of Ophthalmology, Hong Kong Eye Hospital, Hong Kong, PR China; \\ 'Department of Ophthalmology, Grantham Hospital, Hong Kong, PR China
}

\section{Keywords}

ExPRESS mini-shunt $\cdot$ Trabeculectomy $\cdot$ Steroid-induced glaucoma $\cdot$ Pediatric glaucoma

\begin{abstract}
This case report illustrates the use of ExPRESS mini-shunt in a pediatric glaucoma patient. We describe the management of steroid-induced glaucoma with ExPRESS mini-shunt in a 9-yearold boy with allergic keratoconjunctivitis. The intraocular pressure of both of his eyes was uncontrolled with medical and laser treatment. Both eyes were treated with ExPRESS minishunt and mitomycin-C. Transient overfiltration with postoperative hypotony occurred in both eyes and resolved after 2 weeks. One year postoperatively, intraocular pressure was maintained below $21 \mathrm{~mm} \mathrm{Hg}$ without medication in 1 eye. Bleb needling with mitomycin-C was done to maintain filtration. The fellow eye received cataract extraction but developed bleb failure a few months afterwards. The intraocular pressure was controlled medically. To conclude, ExPRESS mini-shunt is a new surgical option in selected patients. Bleb failure developed after cataract extraction. Postoperative inflammation should be minimized. Patient selection, such as those with stable ocular condition, is important to increase surgical success.




\section{Case Reports in Ophthalmology}

\section{Case Report}

A 9-year-old boy was referred by a private ophthalmologist for bilateral steroid-induced glaucoma and cataract in May 2012. He had history of allergic keratoconjunctivitis (AKC) and was on self-prescribed steroid eye drop 3-4 times a day for 1 year. At presentation, his pinhole visual acuity (VA) was 0.3 and 0.2 Snellen decimal in the right eye (RE) and left eye (LE), respectively. Intraocular pressure (IOP) with Goldmann applanation tonometer was 41 $\mathrm{mm} \mathrm{Hg} \mathrm{(RE)} \mathrm{and} 42 \mathrm{~mm} \mathrm{Hg}$ (LE) despite topical dorzolamide, timolol, latanoprost, and oral acetazolamide. His cup-disc ratio (CDR) was 0.9 in the RE, and relative afferent pupillary defect was present. The LE CDR was 0.7. Gonioscopy showed bilateral 360-degree open angles. There was bilateral moderate posterior subcapsular cataract.

The child was uncooperative for automated visual field testing. Optical coherence tomography (OCT) (Spectralis OCT, Heidelberg, Germany) of the retinal nerve fiber layer showed severe generalized thinning in the RE and superotemporal and inferotemporal rim thinning of the retinal nerve fiber layer in the LE (Fig. 1). IOP remained uncontrolled despite maximal topical anti-glaucoma treatment and selective laser trabeculoplasty. AKC symptoms were controlled with topical olopatadine. ExPRESS Miniature Glaucoma DeviceP-50 (Alcon, Neve-Ilan, Israel) was implanted sequentially in the LE superotemporally 3 weeks later and in the RE superonasally 1 month later. The trabeculectomy was conducted according to the standard trabeculectomy procedure. The conjunctiva was dissected and a $3 \times 3$-mm scleral flap was created. Subsequently, $0.4 \mathrm{mg} / \mathrm{ml}$ mitomycin-C (MMC) was placed subconjunctivally and under the scleral flap for $3 \mathrm{~min}$, followed by irrigation with balanced-salt solution. The anterior chamber was then entered under the scleral flap at the grey zone at limbus with a 25-gauge needle. The ExPRESS device was implanted under the scleral flap through the needle track. The scleral flap was closed with 2 stitches of $10 / 0$ nylon suture, while the conjunctiva was closed with $8 / 0$ vicryl suture. The patient was started on pred forte $1 \%$ eye drop every $2 \mathrm{~h}$, and moxifloxacin eye drop 3 times daily postoperatively, taped over 2 months, followed by tapering dose of dexoptic-N eye drop (dexamethasone and neomycin) for another 3 months. There was overfiltration with hypotony (IOP $<6 \mathrm{~mm} \mathrm{Hg}$ ) in the early postoperative period in both eyes, requiring anterior chamber reformation with viscoelastic agent. There were no hypotony-related ocular complications including maculopathy and choroidal effusion. IOP was controlled in the range of $10-19 \mathrm{~mm} \mathrm{Hg}$ without the need for any antiglaucoma medication since 3 weeks postoperation. The filtering blebs remained functional bilaterally, and were small and cystic in RE and diffuse in LE.

Lens aspiration and implantation of intraocular lens (SA60AT+ 19.0D) (Alcon, Fortworth, TX, USA) was performed uneventfully in the RE 4 months after filtration surgery. The patient was started on intensive steroid eye drop loteprednol etabonate (lotemax) every hourly to reduce inflammation postoperatively. Lotemax was chosen over Pred forte to reduce the risk of steroid response. However, IOP in both eyes began to rise above $21 \mathrm{~mm} \mathrm{Hg}$, and occasionally up to above $40 \mathrm{~mm} \mathrm{Hg}$, in the 5 months following the cataract operation, despite the use of multiple anti-glaucoma eye drops. In view of failing blebs, subconjunctival needling with anti-metabolite injection was performed twice in each eye (RE $50 \mathrm{mg} / \mathrm{ml} \mathrm{5-}$ fluorouracil in first episode and $0.2 \mathrm{mg} / \mathrm{ml} \mathrm{MMC} \mathrm{in} \mathrm{second} \mathrm{episode;} \mathrm{LE} 0.2 \mathrm{mg} / \mathrm{ml} \mathrm{MMC} \mathrm{was}$ applied for both episodes). The subconjunctival space was entered with a 27-gauge needle, the subconjunctival adhesion was lysed and the scleral flap was elevated during the needling procedures. However, the anterior chamber was not entered during the presence of the ExPRESS device. IOP remained controlled at $12 \mathrm{~mm} \mathrm{Hg}$ over the RE and $14 \mathrm{~mm} \mathrm{Hg}$ over the LE at 4 months afterwards although his $\mathrm{RE}$ required topical timolol/travoprost for optimal con- 
trol. The LE did not require any anti-glaucoma medications. Fig. 2a, b showed RE underfiltrating bleb and LE functioning bleb, respectively. In the RE, the best-corrected VA remained 0.3 Snellen decimal due to advanced glaucoma, while that in the LE was 0.4 Snellen decimal.

\section{Discussion}

Compared to adults, management of glaucoma in pediatric patients is unique in the surgical difficulties caused by smaller eyes, low scleral rigidity, and thin sclera. Additional challenges lie in the more intense inflammatory response that can cause a higher failure rate in drainage surgery, and difficulties in cooperation for preoperative assessment and postoperative management [1].

ExPRESS ${ }^{\circledR}$ Glaucoma Filtration Device is a small metallic shunt that diverts aqueous from the anterior chamber to the subtenon space. It was chosen over other filtration surgery in this case for several reasons. First, pediatric trabeculectomy is known to have a high rate of failure [1]. Anti-fibrotic agents including MMC have been used to increase bleb survival but the available evidence is conflicting [2]. There was no significant difference in final IOP between groups with conventional trabeculectomy and ExPRESS [3]. However, studies had shown significantly higher complete success rate with ExPRESS compared to trabeculectomy at 1 year [4, 5]. In our case, IOP was well controlled in the early and intermediate postoperative periods. However, IOP control deteriorated in both eyes soon after RE cataract operation. We postulate that late IOP rise was triggered by the postoperative inflammatory response.

Reducing postoperative inflammation was another reason why ExPRESS shunt was chosen over trabeculectomy or glaucoma implant. By avoiding iridectomy and reducing the size of sclerotomy, ExPRESS implantation causes less postoperative inflammation and subsequent scarring at the scleral flap and subconjunctival space, thereby minimizing failure [6]. The RE trabeculectomy with ExPRESS implantation failed after cataract extraction probably due to inflammation. It might be preferable to perform cataract extraction first and wait until the inflammation subsides before proceeding to trabeculectomy.

ExPRESS shunt also reduces surgical difficulty and surgical trauma for small pediatric eyes with shorter operative time. This may explain the quicker recovery of VA at postoperative 1 week in patients with ExPRESS shunt, when compared to 1 month in those with trabeculectomy [7]. Faster visual recovery is essential for children undergoing eye operation in avoiding amblyopia, as well as helping them resume normal learning activities as soon as possible.

Maris et al. [6] identified a lower hypotony rate of 4\% in ExPRESS implantation compared to $32 \%$ in trabeculectomy patients. This is a major concern in pediatric filtration surgery as aqueous may drain more easily with low scleral rigidity and thin flap [8]. It was proposed that the standardized lumen of the ExPRESS device gave a fixed controlled resistance to the flow of aqueous from anterior chamber to the subtenon space [9], thus reducing overfiltration. In addition to the concerns about blinding hypotony-related complications, e.g. corneal decompensation and maculopathy, if choroidal effusion occurs, the use of corticosteroid in a growing child may pose significant systemic side effects. It can also exacerbate steroid-induced glaucoma as in our patient. Although hypotony still occurred in our case, its severity may be reduced by tighter sutures and smaller scleral flap intraoperatively in future cases. Leaving a small amount of viscoelastic agent in the eyes may also help. 
Potential shortcomings of the ExPRESS device in pediatric glaucoma are that the space for insertion of the device is limited by smaller eyes and narrower space in the angle. If the device is not inserted in an ideal plane, it may traumatize the corneal endothelium and iris, causing pigment dispersion that may worsen IOP control. In addition, as the device is planned to be left in the eye for decades in children, there is an increased risk of device erosion, malposition, and dislocation, as active children may be more prone to trauma or simply by ocular growth. Therefore, the ExPRESS device may only be suitable in selected group of pediatric glaucoma patients, such as those with wide-open angle and older age group in which the eyes are relatively more static in size. At the time of writing this case report, we only found 1 other case report on the use of the ExPRESS device in a pediatric patient with Sturge-Weber syndrome [10]. That patient had a good surgical outcome in terms of IOP control at 6 months. However, their technique was different from ours, as they inserted the ExPRESS device subconjunctivally (instead of under the scleral flap) and the surgery was performed in 2 stages (ExPRESS device implantation before repeated trabeculectomy) in an eye with previous trabeculectomy.

Another surgical option is glaucoma drainage device (GDD) implantation. Its surgical success rate was comparable with trabeculectomy, but it decreased to $42-45 \%$ in 4 years' time [11]. Theoretically, a valved GDD has a lower rate of hypotony as the tube only allows aqueous to drain at IOP $>8 \mathrm{~mm} \mathrm{Hg}$. However, hypotony still occurred at a reported rate of $11-24 \%$ [10]. GDD is also associated with its unique tube-related complications, not to mention that it is surgically more demanding than trabeculectomy, making it less than ideal for pediatric glaucoma patients.

In managing pediatric glaucoma patients, we may need special attention in interpreting investigations such as OCT. There is no built-in normative database for pediatric subjects, and thus the OCT finding should be interpreted with care. The trend of changes may be a more important indicator than the absolute value of RNFL thickness. Moreover, specular microscopy for endothelial cell count should be monitored in this child after multiple intraocular surgeries.

\section{Conclusion}

To conclude, ExPRESS device implantation seems to be safe and effective in achieving IOP control in at least short to medium term in pediatric glaucoma patients. It can be considered as an alternative to conventional trabeculectomy. Case selection and avoidance of triggers which may cause shunt failure may enhance success rates in children. Further studies would be needed to establish the long-term safety profile and success rate of its use in pediatric patients.

\section{Statement of Ethics}

The study conformed to the tenets of the Declaration of Helsinki.

\section{Disclosure Statement}

The authors have no financial interests to disclose. 
References

1 Papadopoulos M, Khaw PT: Advances in the management of paediatric glaucoma. Eye 2007;21:13191325.

-2 Tanimoto SA, Brandt JD: Options in pediatric glaucoma after angle surgery has failed. Curr Opin Ophthalmol 2006;17:132-137.

-3 Buys YM: Trabeculectomy with ExPRESS: weighing the benefits and cost. Curr Opin Ophthalmol 2013;24:111-118.

4 de Jong L, Lafuma A, Aguade AS, et al: Five-year extension of a clinical trial comparing the EX-PRESS glaucoma filtration device and trabeculectomy in primary open-angle glaucoma. Clin Ophthalmol 2011;5:527-533.

5 Dahan E, Ben Simon GJ, Lafuma A: Comparison of trabeculectomy and Ex-PRESS implantation in fellow eyes of the same patient: a prospective, randomised study. Eye 2012;26:703-710.

-6 Maris PJ Jr, Ishida K, Netland PA: Comparison of trabeculectomy with Ex-PRESS miniature glaucoma device implanted under scleral flap. J Glaucoma 2007;16:14-19.

7 Good TJ, Kahook MY: Assessment of bleb morphologic features and postoperative outcomes after ExPRESS drainage device implantation versus trabeculectomy. Am J Ophthalmol 2011;151:507-513.

-8 Ou Y, Caprioli J: Surgical management of pediatric glaucoma. Dev Ophthalmol 2012;50:157-172.

-9 Rouse JM, Sarkisian SR Jr: Mini-drainage devices: the Ex-PRESS Mini-Glaucoma Device. Dev Ophthalmol 2012;50:90-95.

10 Elgin U, Simsek T, Batman A: Use of the ex-press miniature glaucoma implant in a child with SturgeWeber syndrome. J Pediatr Ophthalmol Strabismus 2007;44:248-250.

11 Yang HK, Park KH: Clinical outcomes after Ahmed valve implantation in refractory paediatric glaucoma. Eye (Lond) 2009;23:1427-1435.

This case report was previously presented as poster presentation at the Annual Scientific Meeting 2013, organized by the College of Ophthalmologists of Hong Kong in December 2013 
Choy et al.: ExPRESS Mini-Shunt as a Treatment Alternative for Medically Uncontrolled Steroid-Induced Glaucoma in a Pediatric Patient
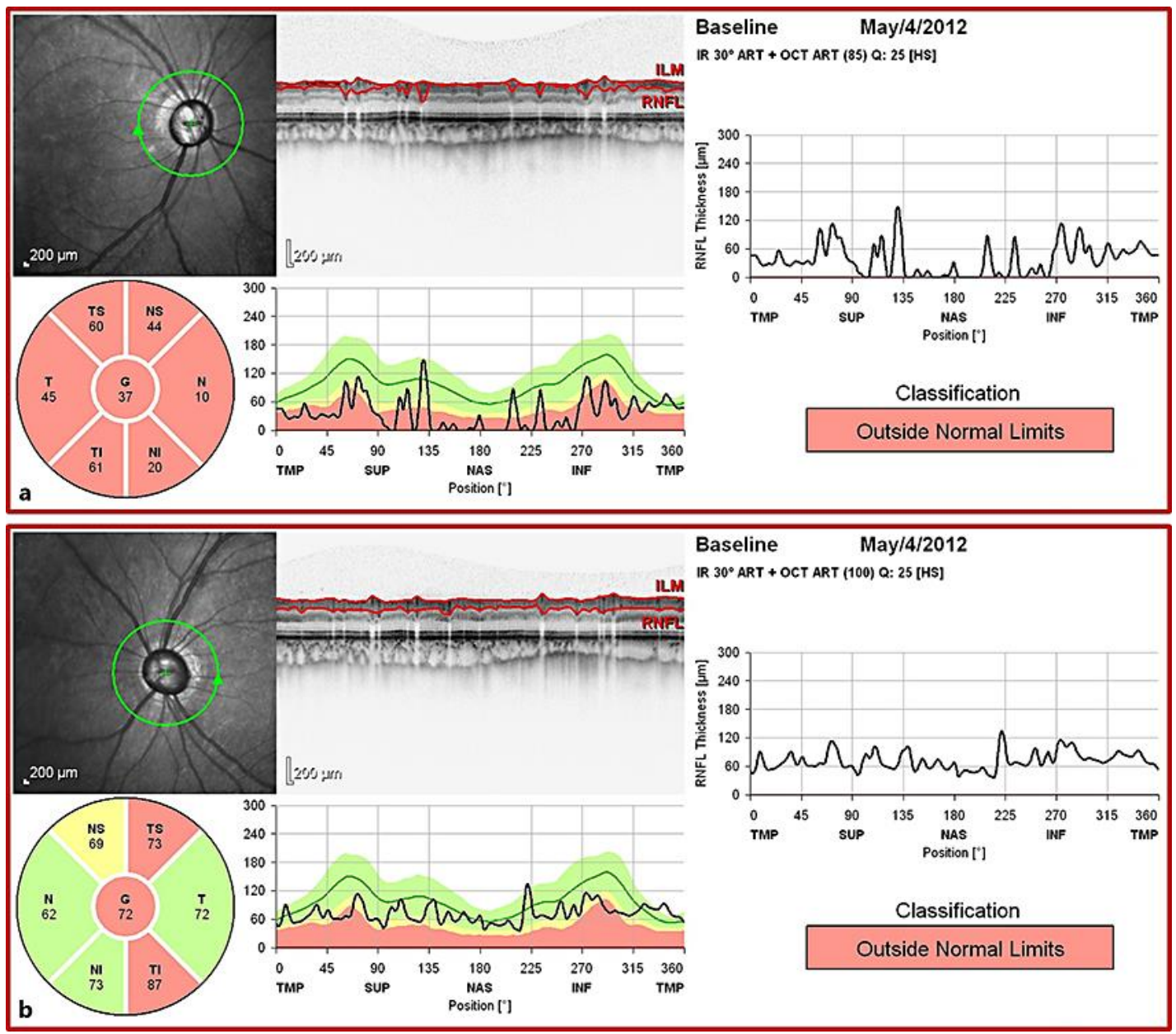

Fig. 1. OCT showing severe generalized thinning in the RE (a) and superotemporal and inferotemporal rim thinning in the LE (b) of the retinal nerve fiber layer on presentation. 


\section{Case Reports in Ophthalmology}

\begin{tabular}{l|l}
\hline Case Rep Ophthalmol 2016;7:270-276 \\
\hline DOI: 10.1159/000453393 & $\begin{array}{l}\text { @ 2016 The Author(s). Published by S. Karger AG, Basel } \\
\text { www.karger.com/cop }\end{array}$ \\
\hline
\end{tabular}

Choy et al.: ExPRESS Mini-Shunt as a Treatment Alternative for Medically Uncontrolled Steroid-Induced Glaucoma in a Pediatric Patient
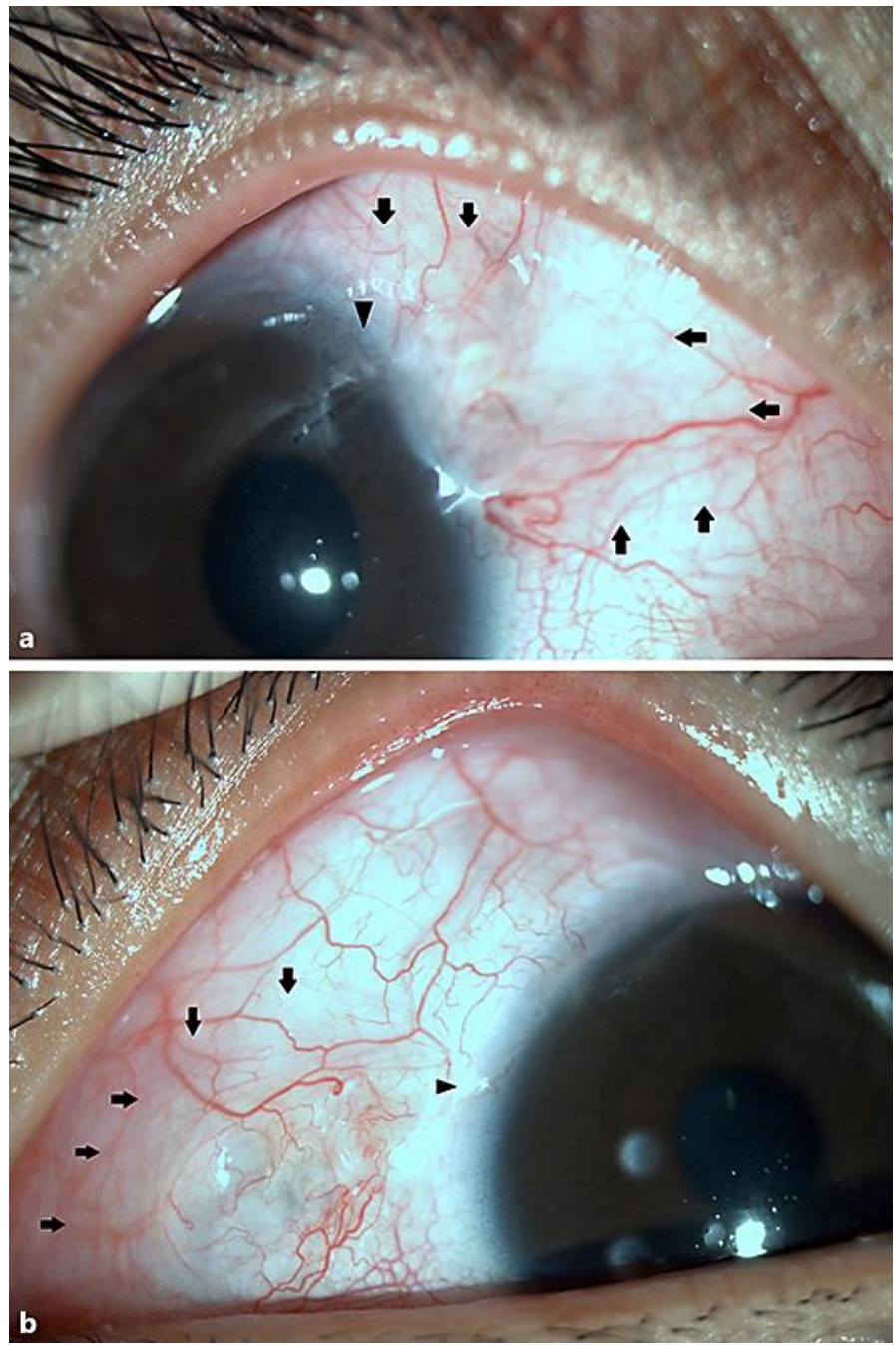

Fig. 2. Underfiltrating bleb in the RE (a) and functioning bleb in the LE (b) at around 5 months postfiltration surgery, with intraocular lens implanted in the RE (the area of the bleb is indicated by arrows, while the position of the ExPRESS shunt is indicated by the arrowhead). 